Abstract

\title{
Cooperative Monocular SLAM for Multi-UAV Systems ${ }^{\dagger}$
}

\author{
Juan-Carlos Trujillo ${ }^{1}$, Rodrigo Munguia ${ }^{1}$, Edmundo Guerra ${ }^{2}$ and Antoni Grau 2,** \\ 1 Department of Computer Science, CUCEI, University of Guadalajara, Guadalajara 44430, Mexico; \\ rodrigo_fma@hotmail.com (J.-C.T.); rodrigo.munguia@upc.edu (R.M.) \\ 2 Department of Automatic Control, Technical University of Catalonia UPC, 08034 Barcelona, Spain; \\ edmundo.guerra@upc.edu \\ * Correspondence: antoni.grau@upc.edu \\ + Presented at the 5th International Symposium on Sensor Science (I3S 2017), Barcelona, Spain, \\ 27-29 September 2017.
}

Published: 4 December 2017

The capacity of autonomous navigation is an important requirement for applications involving micro aerial vehicles (MAVs). In this sense, GPS represents the typical solution for determining the position of a MAV operating in outdoor and open environments. However, for indoor or cluttered environments, GPS is not reliable enough. Also, the magnitude of the GPS error can be unacceptable for applications requiring high precision localization. In this context, the visual-based approaches represent an interesting alternative for addressing the problem of MAV navigation. In particular, the visual SLAM (Simultaneous Localization and Mapping) techniques have received great attention from the research community. Using SLAM, a mobile robot can localize itself using only its on-board sensors while it moves across an unknown environment. This research treats and presents a novel cooperative visual SLAM system that is used for addressing the navigation problem of a team of MAVs operating in GPS-denied environments. In this case, it is shown that using only visual information obtained from monocular cameras equipped on-board of the MAVs, the observability properties of the system can be improved. The above is achieved by incorporating measurements of the relative distance between the MAVs. The experimental results validate that the proposed system provides a good estimation of the position and orientation of each MAV.

(C) 2017 by the authors. Licensee MDPI, Basel, Switzerland. This article is an open access article distributed under the terms and conditions of the Creative Commons Attribution (CC BY) license (http://creativecommons.org/licenses/by/4.0/). 